\title{
Structural basis for the mechanism of ABC transporters
}

Author: Konstantinos Beis ${ }^{1,2,3, *}$

\section{Affiliations:}

${ }^{1}$ Department of Life Sciences, Imperial College London, Exhibition Road, London, South Kensington, SW7 2AZ, United Kingdom.

${ }^{2}$ Membrane Protein Lab, Diamond Light Source, Harwell Science and Innovation Campus, Chilton, Oxfordshire, OX11 0DE, United Kingdom.

${ }^{3}$ Rutherford Appleton Laboratory, Research Complex at Harwell, Didcot, Oxfordshire OX11 0FA, United Kingdom.

*Correspondence to: kbeis@imperial.ac.uk 


\section{Abstract}

The ATP binding cassette (ABC) transporters are primary transporters that couple the energy stored in ATP to the movement of molecules across the membrane. ABC transporters can be divided into exporters and importers; importers mediate the uptake of essential nutrients into cells and are found predominantly in prokaryotes whereas exporters transport molecules out of cells or into organelles and are found in all organisms. ABC exporters have been linked with multi drug resistance in both bacterial and eukaryotic cells. ABC transporters are powered by the hydrolysis of ATP and transport their substrate via the alternating access mechanism, whereby the protein alternates between a conformation in which the substrate binding site is accessible from the outside of the membrane, outward-facing, and one in which it is inward-facing. In this mini-review, the structures of different $A B C$ transporter types in different conformations are presented within the context of the alternating access mechanism and how they have shaped our current understanding of the mechanism of ABC transporters.

keywords: $\mathrm{ABC}$ importer, $\mathrm{ABC}$ exporter, $\mathrm{ABC}$ transporter mechanism, membrane protein structure

\section{Introduction}

$\mathrm{ABC}$ transporters are one of the largest superfamilies and are found among bacteria, archaea and eukaryote [1]. Their functional role is very diverse, ranging from importing essential nutrients inside bacterial cells to conferring multi-drug resistance in cancer cells. ABC transporters can be classified as importers (type I and II) or exporters; type I and II importers are only found in bacteria and their role is to uptake 
essential nutrients whereas exporters transport substrates out of the cell. They have been associated with multi drug resistance in both bacterial and eukaryotic cells. Despite their distinct roles, both importers and exporters require the binding and hydrolysis of ATP for energisation. Their core architecture is a dimeric transmembrane domain (TMD) that contains the ligand binding site and a dimeric nucleotide binding domain (NBD) whereas ATP binds and hydrolyses [2]. Importers have an additional substrate binding protein (SBD) that binds the ligands and transfers them to the specific TMD for transport. The TMD amino acid sequence and topology are not conserved among the different types of $\mathrm{ABC}$ transporters. The only common structural component is the NBDs, with various conserved motifs, which couples ATP binding and hydrolysis to the TMDs whereas significant conformational changes occur for substrate translocation. Importers and exporters use the alternating access mechanism and they alternate between inward- and outward-facing states, which exposes the ligand binding site to the inside or outside of the membrane. Importers accept their substrate from the periplasmic face of the membrane and have to alternate from an outward to inward conformation, whereas exporters transport their substrate from the inside to the outside of the cell and alternate between inward and outward conformations for efflux. In recent years, structures from both importers and exporters have been determined at high resolution and in multiple conformations that have allowed the elucidation of their molecular mechanism. In this mini-review, the general architecture of the transporters and structures in the various conformations are outlined in the context of the alternating-access mechanism.

\section{Nucleotide-Binding Domain}


The NBDs are conserved throughout the different ABC transporter types. They have been called the 'engine’ of the transporter [3] since ATP binding, hydrolysis and release gives rise to the energy needed for the transport of molecules. Each NBD can be divided in two subdomains, a catalytic core domain and an $\alpha$-helical domain. The catalytic core domain contains conserved motifs for the binding and hydrolysis of ATP; the Walker A motif (P-loop) interacts with the phosphate groups of the nucleotide and the Walker B motif has a glutamate residue that acts as a general base to activate a water molecule for nucleophilic attack at the $\gamma$-phosphate of ATP. The $\alpha$ helical domain contains the motif LSGGQ known as the ABC-signature motif (or Cloop) that is involved the binding of the nucleotide. The NBDs are arranged "head-totail” that form the full composite ATP-binding and hydrolysis site upon dimerization via interactions with the D-loop; the ATP binding site is formed from the Walker A and Walker B motifs of one subunit and the Q-loop of the other subunit, so that two ATP molecules can bind and hydrolyze. In the absence of nucleotides, the NBDs are separated, open conformation, but upon ATP binding they come close together to sandwich the ATP molecules and form the complete interface. ATP hydrolysis disrupts the dimer interface and releases ADP and inorganic phosphate. In heterodimeric ABC-exporters ATP hydrolysis is asymmetric and the NBDs are not completely dissociated since one NBD can hydrolyze ATP (consensus site) whereas the other one is very slow (degenerate site) as a result of Walker B mutations; the only full length heterodimeric ABC exporter structure is the TM287/288 from Thermotoga maritime $[4,5]$ in the presence and absence of nucleotides which demonstrates how the NBDs interact with the ATP and its implications in the transport cycle. 


\section{Coupling helices}

Effective coupling of transport to ATP binding requires the transmission of the molecular motion from the NBDs to the TMDs. The TMDs of both importers and exporters interacts with the NBDs through an $\alpha$-helix, coupling helix, that is located in the cytoplasmic loops of the TMD. The structure of the coupling helix is conserved but its sequence varies between the different transporters. In exporters, such as Sav1866 [6] and P-glycoprotein (P-gp) [7], and importers, [8] that contain six TM helices, the coupling helix is between TM4 and TM5. In the maltose transporter MalFGK $_{2}$ the coupling helices are between TM6 and TM7 in MalF and TM4 and TM5 in MalG [9]. The BtuCD contains ten TM helices and the coupling helix is between TM6 and TM7 [10].

\section{Type I ABC importers}

The maltose transporter MalEFGK ${ }_{2}$ from Escherichia coli has been crystallized in various conformations which have revealed the detailed mechanism for type I importers (Figure 1). The maltose transporter consists of a periplasmic SBD, maltose binding domain, (MPB, MalE) that binds maltose, two TMDs (MalF and MalG) and two NBDs (MalK) [9]. In the maltose transporter MalFGK 2 the transmembrane region consists of two subunits: MalF with eight TM helices and MalG with six TM helices. The TM helices 1-3 of MalF contact TM helices $2-4$ of MalG, and TM helix 1 of MalG packs against the helix bundle formed by TM helices 4-8 of MalF. MalF has an extended periplasmic domain between the TM helices 3 and 4. MalF TM helices 3-8 and MalG TM helices 1-6 have similar topology and are related by an approximate two-fold axis perpendicular to the membrane bilayer. The structure of the maltose 
transporter has been solved in the presence of maltose and larger sugars that provide key insights in the recognition and transport mechanism of type I importers [9, 11]. A large cavity is formed at the interface of MBP, MalF and MalG that reaches halfway across the membrane bilayer that binds a maltose molecule [9]. Two aromatic residues stack against the carbohydrate rings, as is frequently observed at sugar binding sites in proteins, and there are several hydrogen bonds. In the crystal structure, the MBP does not have maltose bound and is in an open conformation, implying that it has delivered the maltose to the transporter.

In its resting state, the maltose transporter adopts an inward-facing conformation with its substrate binding cavity open to the cytoplasm; the MalF and MalG subunits form a periplasmic gate that shields the transporter, whereas they have separated at the cytoplasmic side and exposed the binding cavity [12] (Figure 1). In the absence of nucleotides, the two NBDs, MalK, are also in an open conformation. The transport cycle starts when maltose loaded MalE binds to the TMD and adopts the pretranslocation state with movement of the TM helices [13]. The MalF and MalG subunits form an occluded maltose binding cavity that is sealed by periplamic and cytoplasmic gates. The pre-translocation state is an intermediate between the inwardand outward-facing states. As a result of these domain movements, the two MalK subunits come closer to each other that can bind ATP. Binding of ATP, results in dimerization of the two MalK subunits, and conformational changes along the TMD and MBP; the transporter adopts an outward-facing conformation whereas the periplasmic gate opens (but remains sealed by the bound MBP) and a closed cytoplasmic gate is formed [14]. Conformational changes along the TMD, open the MPB that releases maltose inside the TMD binding cavity [9]. ATP hydrolysis 
releases maltose from the cavity, MBP dissociates from the MalFG subunits and the transporter resets in its inward-facing state for another cycle.

\section{Type II ABC importers}

The best structurally characterized type II ABC importer is the vitamin $\mathrm{B}_{12}$ transporter from E. coli in different conformations (Figure 2). The structures of the HiF protein (Hi1470/71) from Haemophilus influenzae [15] and the heme transporter HmuUV from Yersinia pestis [16] have also been reported. The vitamin $\mathrm{B}_{12}$ transporter consists of two TMD subunits (BtuC), two NBDs (BtuD) and a SBD (BtuF) [10]. The main difference between the type II and type I importers is the number of TM helices that span the membrane; each BtuC subunit contains 10 TM helices rather than 5 in type I importers. The BtuC TM helices pack together [17] unlike ABC exporters that intertwine [6]. TM2 is positioned through the center of the subunit in proximity to the other helices, thus dividing the TMD in front and back faces. A substrate translocation pathway is formed at the dimerization interface of the two BtuC subunits; TM 5 and 10 pack against TM 10 and 5 from the opposite subunit. The four TMs form a welldefined cavity that is aligned with residues from both TM 5 and 10 as well as residues from TM 3 and 8. Periplasmic and cytoplasmic gates (gates I and II) determine the accessibility of the cavity by the substrate that adopts different conformations along the transport cycle [18]. The structure of BtuCD-F has been determined in various conformations including the presence or absence of the BtuF subunit and/or nucleotides. To-date, no type II importer has been reported in the presence of a ligand in order to explain how the substrate binds in the TMD and transported. 
The transport cycle starts with the binding of ATP to the outward-open BtuCD [17], which results in closure of the BtuD dimer [19] (Figure 2). The cytoplasmic gate I opens and gate II closes as a result of the conformational changes associated with the BtuD dimerization. BtuF loaded with vitamin $\mathrm{B}_{12}$ binds to the BtuCD, which results in an occluded conformation [18], and subsequent release of vitamin $\mathrm{B}_{12}$ in a lowaffinity cavity. ATP hydrolysis disrupts the BtuD dimer interface and the coupling helices and cytoplasmic gate II open. These conformational changes lead to an inward-facing conformation (represented by the HI1470/71 transporter structure [15]) whereas vitamin $\mathrm{B}_{12}$ can be released in the cytoplasm. Upon release of vitamin $\mathrm{B}_{12}$, the cytoplasmic gate I adopts an asymmetric closed state [10]. Finally, BtuF can dissociate from the BtuCD that results in the rearrangement of cytoplasmic gate I to a symmetric state, which in turn causes the transporter to adopt an outward-open conformation to commence a new transport cycle.

\section{ABC exporters}

Bacterial ABC exporters can exist as homodimers or heterodimers with each monomer composed of a TMD consisting of six TM helices, which forms the translocation pathway across the membrane bilayer and an NBD where the hydrolysis of ATP takes place (Figure 3). The core architecture of some eukaryotic ABC exporters can be built from one chain polypeptide. Unlike ABC importers that have provided structures for an almost complete transport cycle from the same protein, no ABC exporter from the same protein has been trapped in "all” available states to provide a detailed mechanism. Combination of structures from different proteins has elucidated their molecular mechanism, which is supported by biochemical and biophysical data. 
The crystal structure of several bacterial ABC exporters in different conformations has been elucidated: MsbA, lipid A flippase, from E. coli, Salmonella enterica and Vibrio cholera [20], Sav1866 from Staphylococcus aureus [6], NaAtm1 from Novosphingobium aromaticivorans DSM 12444 [21] and McjD, antibacterial peptide self-immunity, from E. coli [22]. The crystal structure of the heterodimeric bacterial ABC exporter TM287-TM288 from T. maritime has also been reported in the presence and absence of nucleotides in an inward-facing conformation [4, 5]. Despite the clinical importance of human ABC transporters, only one structure has been reported, ABCB10, that is involved in erythropoiesis [23]. Eukaryotic structures homologous to human ABC transporters have been elucidated including P-gp from mouse [7, 24] and Caenorhabditis elegans [25], Atm1 from Saccharomyces cerevisiae [26] and from Cyanidioschyzon merolae [27]. All eukaryotic ABC exporters have been crystallized in the same inward-facing state.

The structures of Sav1866 and MsbA [6, 20] in the outward-open nucleotide bound conformation display intertwining of their TM helices unlike ABC importers that pack against each other; the TMDs intertwine to form two 'wings', which are formed by the TMs 1 and 2 of one subunit and TMs 3-6 of the other subunit. The orientation of the TMs forms an open substrate binding site to the periplasm. The structure of the nucleotide bound McjD revealed no intertwining of the TMD [22] unlike Sav1866 and MsbA; it does not display any 'wings' and TM1 is closer to TM6 of the same monomer and to TM1 of the adjacent monomer, while TM2 comes to close proximity of TM5 from the adjacent monomer and TM5 bends towards TM2. This configuration of TMs has resulted to an inaccessible substrate-binding cavity from either the cytoplasmic or the periplasmic face. MsbA has also been crystallized in two inwardfacing conformations, inward open and inward-closed, in the absence of nucleotides 
[20]; the inward-open conformation displays a very wide-open TMD, whereas in the inward-closed conformation, the TMDs are closer to each other while the NBDs remain separate. In both the inward-facing conformations, the TMDs form two 'wings' between TMs 4 and 5 of one subunit and TMs 1-3 and 6 of the other subunit. Similarly to the outward open conformation, a substrate binding site accessible from the cytoplasm is formed.

The only structural information between an ABC exporter and its substrate is from the crystal structure of the bacterial NaAtm1 [21] and eukaryotic Atm1 from S. cerevisiae [26] in complex with glutathione derivatives. The structure is in an inward-facing conformation and TMs 4-6 provide key interactions with the glutathione derivatives. The structure of the mouse P-gp has been determined in complex with cyclic peptide inhibitors [7, 24]; these peptides are bound in different sites within the TMD, which provide useful information on the broad selectivity and multi drug nature of eukaryotic ABC exporters.

The structures of MsbA, Sav1866 and McjD in distinct conformations and in the presence or absence of nucleotides have revealed the molecular mechanism of ABC exporters (Figure 3). Binding of the substrate brings the TM helices close to each other [21] and stimulates ATP binding. In the inward-facing apo conformation the NBDs are separated and binding of ATP brings them together, which in turn causes major conformational changes resulting in the outward-open conformation and release of the substrate $[6,20]$. ATP hydrolysis resets the transporter back to the inwardfacing conformation. This model is widely accepted but how the transporter alternates between the two conformations has been unclear. The structure of McjD in the nucleotide bound outward occluded conformation provided key mechanistic details on 
this process [22]. McjD has structural similarities to the periplasmic closed end of the TMs of apo MsbA (inward-closed), and the cytoplasmic closed side of the nucleotide bound Sav1866 and MsbA (outward-open) [6, 20]. Therefore, McjD is in an intermediate state between the two conformations and it has been proposed that after the ligand has left the outward open cavity, TMs 1-2 rotate away from TMs 3-6 of the opposite monomer to form the outward occluded state without TMD intertwining [22]. Transition from the outward occluded state to the inward open facing conformation will require ATP hydrolysis, which disrupts the NBD dimerization interface, and induces intertwining of TMs. An occluded conformation may also exist in the transition from inward- to outward-facing states.

\section{Concluding remark}

In the past few years we have seen an increase of available ABC transporter structures. The mechanism of bacterial ABC importers is almost complete due to the available structures from the same protein. On the other hand, the mechanism of ABC exporters is somehow less clear and especially with respect to ligand recognition. At the moment we have a composite picture about their mechanism from the different $\mathrm{ABC}$ exporter structures but the question that still remains is if all of them have the same mechanism. For example, the antibacterial peptide ABC exporter McjD appears very specific to its peptide, so will the transport cycle be completely identical to the multi-drug ABC exporters MsbA or Sav1866? In that respect we need to trap more $\mathrm{ABC}$ exporter structure of the same family in different conformations. At the moment, we have a lack of available ABC exporter structures in the presence of ligands. The Atm1 proteins with substrates and P-gp with inhibitors have shed some light on the recognition mechanism of ligands but the field requires a major effort to 
determine structures in the presence of their native ligands similar to the maltose transporter. Such structures will allow the detailed understanding of the mechanism of this important family of transporters.

\section{References}

1 Holland, B., Cole, S. P. C., Kuchler, K. and Higgins, C. F. (2003) ABC Proteins From Bacteria to Man. Academic Press

2 Rees, D. C., Johnson, E. and Lewinson, O. (2009) ABC transporters: the power to change. Nat Rev Mol. Cell Biol. 10, 218-227

3 Locher, K. P. (2009) Review. Structure and mechanism of ATP-binding cassette transporters. Philos. Trans. R. Soc. Lond. B Biol. Sci. 364, 239-245

4 Hohl, M., Briand, C., Grutter, M. G. and Seeger, M. A. (2012) Crystal structure of a heterodimeric $\mathrm{ABC}$ transporter in its inward-facing conformation. Nat. Struct. Mol. Biol. 19, 395-402

5 Hohl, M., Hurlimann, L. M., Bohm, S., Schoppe, J., Grutter, M. G., Bordignon, E. and Seeger, M. A. (2014) Structural basis for allosteric cross-talk between the asymmetric nucleotide binding sites of a heterodimeric ABC exporter. Proc. Natl. Acad. Sci. U S A. 111, 11025-11030

6 Dawson, R. J. and Locher, K. P. (2006) Structure of a bacterial multidrug ABC transporter. Nature. 443, 180-185

$7 \quad$ Aller, S. G., Yu, J., Ward, A., Weng, Y., Chittaboina, S., Zhuo, R., Harrell, P. M., Trinh, Y. T., Zhang, Q., Urbatsch, I. L. and Chang, G. (2009) Structure of Pglycoprotein reveals a molecular basis for poly-specific drug binding. Science. 323, 1718-1722 
8 Gerber, S., Comellas-Bigler, M., Goetz, B. A. and Locher, K. P. (2008)

Structural basis of trans-inhibition in a molybdate/tungstate ABC transporter. Science. 321, 246-250

9 Oldham, M. L., Khare, D., Quiocho, F. A., Davidson, A. L. and Chen, J. (2007) Crystal structure of a catalytic intermediate of the maltose transporter. Nature. 450, $515-521$

10 Hvorup, R. N., Goetz, B. A., Niederer, M., Hollenstein, K., Perozo, E. and Locher, K. P. (2007) Asymmetry in the structure of the ABC transporter-binding protein complex BtuCD-BtuF. Science. 317, 1387-1390

11 Oldham, M. L., Chen, S. and Chen, J. (2013) Structural basis for substrate specificity in the Escherichia coli maltose transport system. Proc. Natl. Acad. Sci. U S A. $110,18132-18137$

12 Khare, D., Oldham, M. L., Orelle, C., Davidson, A. L. and Chen, J. (2009) Alternating access in maltose transporter mediated by rigid-body rotations. Mol. Cell. 33, 528-536

13 Oldham, M. L. and Chen, J. (2011) Crystal structure of the maltose transporter in a pretranslocation intermediate state. Science. 332, 1202-1205

14 Oldham, M. L. and Chen, J. (2011) Snapshots of the maltose transporter during ATP hydrolysis. Proc. Natl. Acad. Sci. U S A. 108, 15152-15156

15 Pinkett, H. W., Lee, A. T., Lum, P., Locher, K. P. and Rees, D. C. (2007) An inward-facing conformation of a putative metal-chelate-type ABC transporter. Science. 315, 373-377

16 Woo, J. S., Zeltina, A., Goetz, B. A. and Locher, K. P. (2012) X-ray structure of the Yersinia pestis heme transporter HmuUV. Nat. Struct. Mol. Biol. 19, 13101315 
17 Locher, K. P., Lee, A. T. and Rees, D. C. (2002) The E. coli BtuCD structure: a framework for ABC transporter architecture and mechanism. Science. 296, 10911098

18 Korkhov, V. M., Mireku, S. A. and Locher, K. P. (2012) Structure of AMPPNP-bound vitamin B12 transporter BtuCD-F. Nature. 490, 367-372

19 Korkhov, V. M., Mireku, S. A., Veprintsev, D. B. and Locher, K. P. (2014) Structure of AMP-PNP-bound BtuCD and mechanism of ATP-powered vitamin B12 transport by BtuCD-F. Nat. Struct. Mol. Biol. 21, 1097-1099

20 Ward, A., Reyes, C. L., Yu, J., Roth, C. B. and Chang, G. (2007) Flexibility in the ABC transporter MsbA: Alternating access with a twist. Proc. Natl. Acad. Sci. U S A. $104,19005-19010$

21 Lee, J. Y., Yang, J. G., Zhitnitsky, D., Lewinson, O. and Rees, D. C. (2014) Structural basis for heavy metal detoxification by an Atm1-type ABC exporter. Science. 343, 1133-1136

22 Choudhury, H. G., Tong, Z., Mathavan, I., Li, Y., Iwata, S., Zirah, S., Rebuffat, S., van Veen, H. W. and Beis, K. (2014) Structure of an antibacterial peptide ATP-binding cassette transporter in a novel outward occluded state. Proc. Natl. Acad. Sci. U S A. 111, 9145-9150

23 Shintre, C. A., Pike, A. C., Li, Q., Kim, J. I., Barr, A. J., Goubin, S., Shrestha, L., Yang, J., Berridge, G., Ross, J., Stansfeld, P. J., Sansom, M. S., Edwards, A. M., Bountra, C., Marsden, B. D., von Delft, F., Bullock, A. N., Gileadi, O., BurgessBrown, N. A. and Carpenter, E. P. (2013) Structures of ABCB10, a human ATPbinding cassette transporter in apo- and nucleotide-bound states. Proc. Natl. Acad. Sci. U S A. 110, 9710-9715 
24 Szewczyk, P., Tao, H., McGrath, A. P., Villaluz, M., Rees, S. D., Lee, S. C., Doshi, R., Urbatsch, I. L., Zhang, Q. and Chang, G. (2015) Snapshots of ligand entry, malleable binding and induced helical movement in P-glycoprotein. Acta Crystallogr. D Biol. Crystallogr. 71, 732-741

25 Jin, M. S., Oldham, M. L., Zhang, Q. and Chen, J. (2012) Crystal structure of the multidrug transporter P-glycoprotein from Caenorhabditis elegans. Nature. 490, $566-569$

26 Srinivasan, V., Pierik, A. J. and Lill, R. (2014) Crystal structures of nucleotide-free and glutathione-bound mitochondrial ABC transporter Atm1. Science. $343,1137-1140$

27 Kodan, A., Yamaguchi, T., Nakatsu, T., Sakiyama, K., Hipolito, C. J., Fujioka, A., Hirokane, R., Ikeguchi, K., Watanabe, B., Hiratake, J., Kimura, Y., Suga, H., Ueda, K. and Kato, H. (2014) Structural basis for gating mechanisms of a eukaryotic P-glycoprotein homolog. Proc. Natl. Acad. Sci. U S A. 111, 4049-4054

\section{Figure legends}

Figure 1. Structure and mechanism of the type I maltose ABC importer MalEFGK 2 . The TMD of the maltose transporter consists of the MalF and MalG subunits which are shown as red and blue ribbons respectively. The NBD, MalG, is shown in green and orange ribbons. MBP (MalE) is shown in magenta. Nucleotide analogues are shown as black sticks. Maltose is shown as yellow spheres. The maltose transporter cycle has been determined from the available crystal structures (see type I ABC importers section). 
Figure 2. Structure and mechanism of the type II vitamin $B_{12} A B C$ importer BtuCDF. The TMD, BtuC, is shown as blue and red ribbons. The BtuD is the NBD and shown in green and orange ribbons. The SBD, BtuF, subunit is shown as magenta. Nucleotide analogues are shown as black sticks. The BtuCDF has been trapped in various conformations that have revealed its molecular mechanism (see type II ABC importers section). BtuCDF has not been trapped in an inward-facing conformation.

Figure 3. Structure and mechanism of bacterial ABC exporters. Sav1866, MsbA and McjD are half transporters; each monomer is shown in blue and red ribbons. Nucleotide analogues are shown as black sticks. ABC exporters alternate between inward- and outward- conformations driven by ATP binding and hydrolysis (see ABC exporters section). 


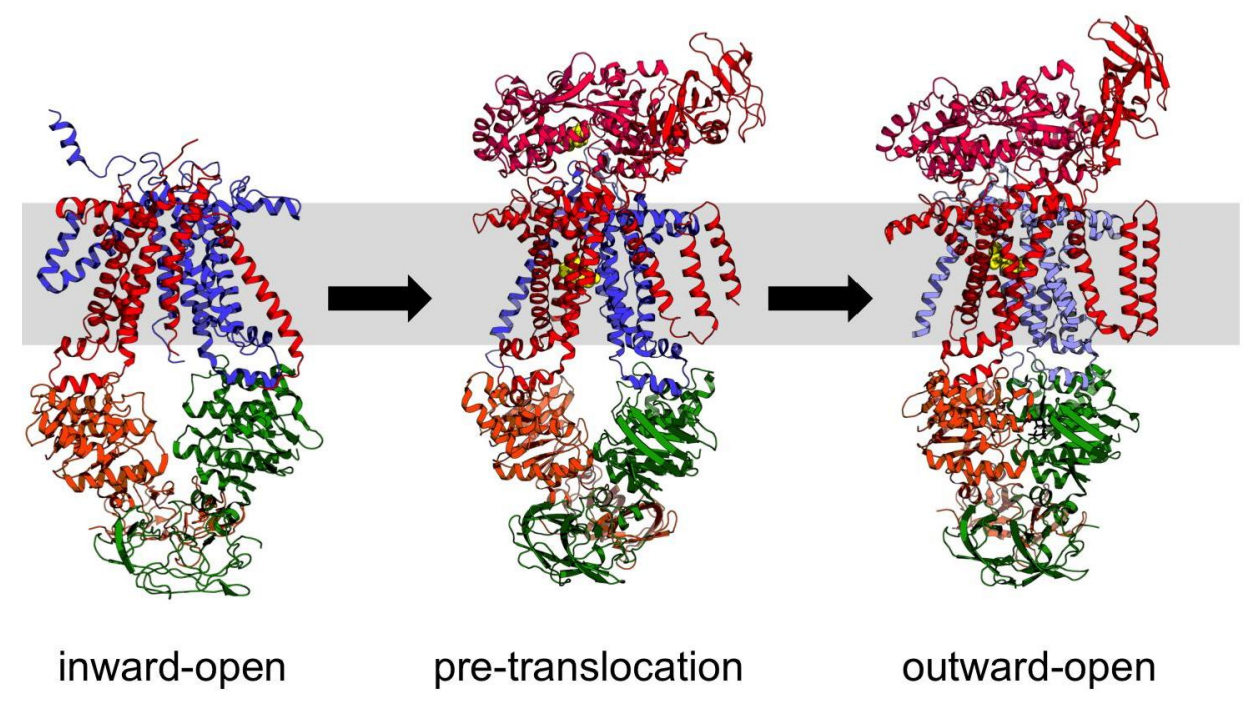




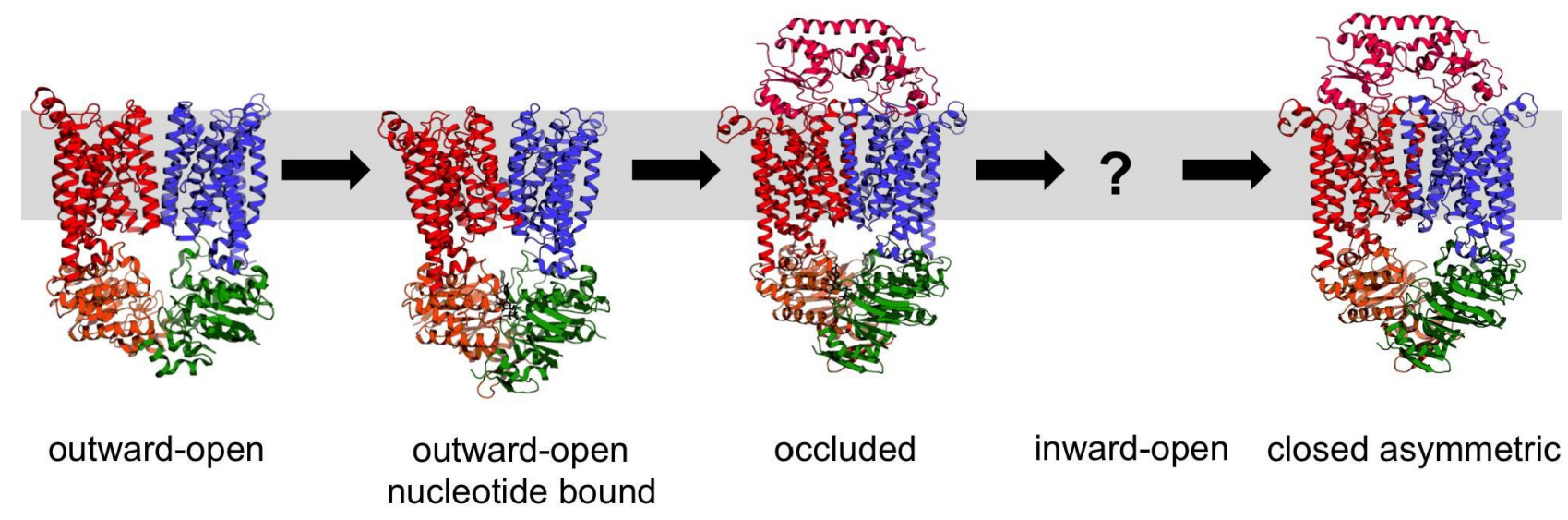




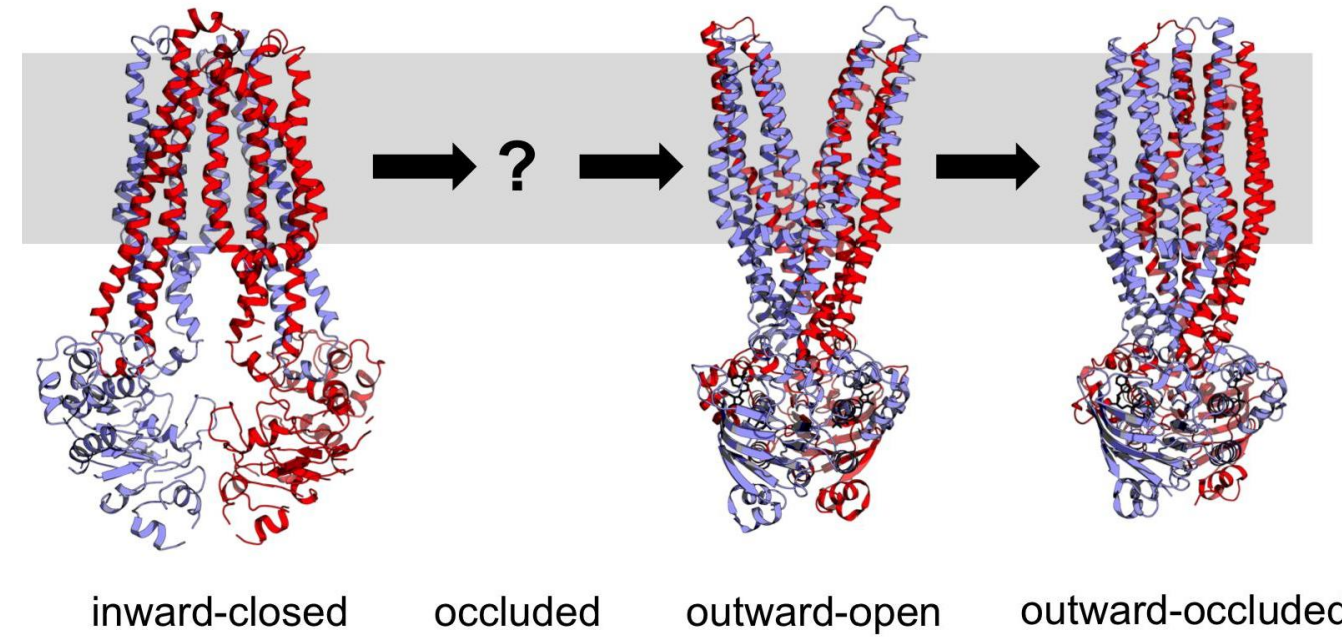

